\title{
How input noise limits biochemical sensing in ultrasensitive systems
}

\author{
Bo Hu, ${ }^{1}$ Wouter-Jan Rappel, ${ }^{2}$ and Herbert Levine ${ }^{3}$ \\ ${ }^{1}$ IBM T.J. Watson Research Center, P.O. Box 218, Yorktown Heights, New York 10598, USA \\ ${ }^{2}$ Center for Theoretical Biological Physics, University of California San Diego, La Jolla, California 92093-0319, USA \\ ${ }^{3}$ Center for Theoretical Biological Physics, Rice University, Houston, Texas 77005, USA
}

(Received 6 April 2014; published 5 September 2014)

\begin{abstract}
Many biological processes are regulated by molecular devices that respond in an ultrasensitive fashion to upstream signals. An important question is whether such ultrasensitivity improves or limits its ability to read out the (noisy) input stimuli. Here, we develop a simple model to study the statistical properties of ultrasensitive signaling systems. We demonstrate that the output sensory noise is always bounded, in contrast to earlier theories using the small noise approximation, which tends to overestimate the impact of noise in ultrasensitive pathways. Our analysis also shows that the apparent sensitivity of the system is ultimately constrained by the input signalto-noise ratio. Thus, ultrasensitivity can improve the precision of biochemical sensing only to a finite extent. This corresponds to a new limit for ultrasensitive signaling systems, which is strictly tighter than the Berg-Purcell limit.
\end{abstract}

DOI: 10.1103/PhysRevE.90.032702

PACS number(s): 87.16.Xa, 02.50.Le, 05.65.+b, 87.23.Kg

\section{INTRODUCTION}

A wide variety of biological processes are controlled by switchlike sensors that are highly sensitive to specific stimuli. For example, Escherichia coli chemotaxis is driven by multiple flagellar motors, which spin clockwise or counterclockwise under the regulation of CheY-P. Recent experiments revealed that bacterial motors exhibit an ultrasensitive response (with a Hill coefficient of $\sim 10$ ) to CheY-P concentrations [1]. Another example is the mitogen-activated protein kinase (MAPK) cascade, a well-conserved signaling module controlling cell fate decisions [2,3]. For instance, the MAPK pathway in Xenopus oocytes converts the concentration of specific hormones into an all-or-none response (oocyte maturation), with a Hill coefficient of at least 35 as estimated in Ref. [3]. Obviously, this ultrasensitivity allows small changes in the input cues to induce dramatic functional effects. As biochemical signals often fluctuate over time due to inherent stochasticity, signaling noise poses a limit to the capacity of concentration sensing. Does ultrasensitivity help the system to read out the input signal? Or does it amplify the input noise to the extent that it corrupts the precision of concentration measurement? What are the general constraints for biochemical sensing? These are the key questions we attempt to address here.

There has been significant interest to understand how signaling noise limits the accuracy of biochemical sensing [4-16]. In 1977, Berg and Purcell argued that the physical limit to concentration measurements is set by the dynamics of their random arrival at target locations [4]: For a single sensor of linear size $a$, the precision of concentration measurements is $\delta c / c \sim 1 / \sqrt{D a c T}$, where $c$ is the concentration of the molecules interacting with the sensor, $D$ is the diffusion constant of the molecules, and $T$ is the measurement time. The Berg-Purcell limit was later generalized to an array of sensors $[5,6]$ and the precision of biochemical sensing was again found to be limited by the molecular counting noise, independent of the number or the sensitivity of sensors. More recent studies have extended the problem of concentration sensing to more sophisticated tasks such as spatial and temporal gradient sensing [8-12] and have explored possible mechanisms that beat the Berg-Purcell limit $[14,15]$.
The interplay between ultrasensitivity and noise is intriguing, as small variations in the input may cause large output differences. However, the nonlinearity of ultrasensitive systems makes theoretical progress difficult. Previous studies usually assume that the fluctuation is small such that one can linearize the input noise in the chemical Langevin equation [17-19] or in the fluctuation-dissipation analysis [20]. This small noise approximation allows for analytical treatment but may not correctly capture the impact of noise or the sensing capacity of ultrasensitive systems. In this paper, we present a simple model consisting of multiple ultrasensitive sensors that measure a (noisy) input signal. We explicitly derive the upper and lower bounds for the output sensory noise. In contrast to the additive noise rule derived earlier $[17,18,20]$, our result shows that the output noise is strictly bounded. We further show that the apparent sensitivity of the system is also constrained by the input signal-to-noise ratio. As a result, we find a fundamental limit to biochemical sensing for arbitrarily ultrasensitive systems. This new limit is strictly tighter than the Berg-Purcell limit and can be applied to both Poisson and non-Poisson input signals.

\section{MODEL}

The input of our model refers to a biochemical signal, $X(t)$, which is fluctuating over time around a mean level. The input fluctuations may arise from, for example, the random birth (synthesis) and death (decomposition) of molecules. Without loss of generality, this input can be described by the following Langevin equation [21]:

$$
\frac{d X(t)}{d t}=-\frac{X(t)-c}{\tau_{x}}+\eta(X, t),
$$

where the parameter $\tau_{x}$ sets the time scale over which the input signal reverts to its mean level $c$. To prevent $X(t)$ from being negative [21], the stochastic term in Eq. (1) is assumed to satisfy $\langle\eta(X, t)\rangle=0$ and $\left\langle\eta(X, t) \eta\left(X^{\prime}, t^{\prime}\right)\right\rangle=\sigma^{2} X(t) \delta\left(t-t^{\prime}\right)$. In steady state, the input signal is found to follow the Gamma distribution:

$$
p(X=x)=\frac{\beta^{\alpha} x^{\alpha-1} e^{-\beta x}}{\Gamma(\alpha)},
$$


with the shape parameter $\alpha \equiv 2 c /\left(\tau_{x} \sigma^{2}\right)$ and the rate parameter $\beta \equiv 2 /\left(\tau_{x} \sigma^{2}\right)$. By Eq. (2), the stationary variance of $X(t)$ is given by $\sigma_{x}^{2}=c \tau_{x} \sigma^{2} / 2$ and thus the Fano factor is simply $\sigma_{x}^{2} / c=1 / \beta$ (the scale parameter). By tuning $\tau_{x}$ or $\sigma$, Eq. (1) can be used to describe both Poisson $(\beta=1)$ and non-Poisson $(\beta \neq 1)$ fluctuations. We also observe that $\alpha=\beta c=c^{2} / \sigma_{x}^{2}$. So the shape parameter $\alpha$ can be interpreted as the signal-to-noise ratio. For most biological systems, it is expected that $\alpha \gg 1$ and hence the zero point is inaccessible, i.e., $p(X=0)=0$, by Eq. (2). As a common example, the input signal $X(t)$ may refer to the number of molecules diffusing in an open volume. This can be described in our model by setting $\beta=1$ (Poisson noise) such that $\alpha=c$, which denotes the average number of molecules in the volume.

The output of our model contains $N$ identical receptors, which independently bind the chemical ligands and switch between the on and off states. As a first step, we assume that these receptors are so close to each other in space that they experience the same local concentration. In this scenario, the effect of ligand diffusion is negligible. Since all the receptors are regulated by the same noisy input signal, they can be correlated. A good example could be multiple bacterial motors under the regulation of CheY-P in the same E. coli. Given the small size of bacteria, it could be a reasonable approximation to assume that all the motors experience the same CheY-P signal. In our general model, the switching process is governed by $X(t)$ through the input-dependent transition rates $k_{+}(X)$ and $k_{-}(X)$, which may be inferred from experiments. We denote the state of the $i$ th sensor by $Y_{i}(t)$, which equals 1 (or 0 ) for the on (or off) state at time $t$. In many biological sensory systems, the input-output relationship exhibits ultrasensitivity and can be described by a Hill equation. In the deterministic case, this means

$$
\lim _{\sigma_{x} \rightarrow 0}\left\langle Y_{i}\right\rangle \equiv f(X=c)=\frac{k_{+}(c)}{k_{+}(c)+k_{-}(c)}=\frac{c^{h}}{c^{h}+K_{d}^{h}},
$$

where $h$ is the Hill coefficient describing the degree of the sensitivity and $K_{d}$ is the dissociation constant at which $f\left(K_{d}\right)=$ $1 / 2$. Equation (3) constrains the possible forms of $k_{ \pm}(X)$. For example, if the sensor has a constant off rate $k_{-}=1 /\left(2 \tau_{y}\right)$, then the on rate has to be $k_{+}(X)=\left(2 \tau_{y}\right)^{-1}\left(X / K_{d}\right)^{h}$. This can be a coarse-grained model for the cooperative binding of transcriptional factors to a DNA promoter [19]. Another possible scheme is $k_{+}(X)=\tau_{y}^{-1} f(X)$ and $k_{-}(X)=\tau_{y}^{-1}[1-f(X)]$, which is appropriate for modeling the cooperativity of bacterial flagellar motors [22,23]. Different phenomenological forms of $k_{ \pm}(X)$ have been studied in the model but do not alter our main results or conclusions given that the receptor time scale is sufficiently short. For convenience, we only report the results for the case of $k_{+}(X)=\left(2 \tau_{y}\right)^{-1}\left(X / K_{d}\right)^{h}$ and $k_{-}=\left(2 \tau_{y}\right)^{-1}$, which at $h=1$ recovers the model we recently solved [21].

In the deterministic case $\left(\sigma_{x} \rightarrow 0\right)$, each sensor follows the simple telegraph process, which has an autocorrelation time equal to $1 /\left[k_{+}(X)+k_{-}\right]=\tau_{y}$ at $X=K_{d}$. Thus, $\tau_{y}$ sets the sensor's time scale and is assumed to be much shorter than the input correlation time, $\tau_{x}$. Only in this scenario can the (slow) input noise appreciably affect the switching statistics of (fast) sensors [17,18,21-24]. The presence of input noise will change the observed input-output relationship, making the average output $\left\langle Y_{i}\right\rangle$ deviate from $f(\langle X\rangle=c)$, as shown in
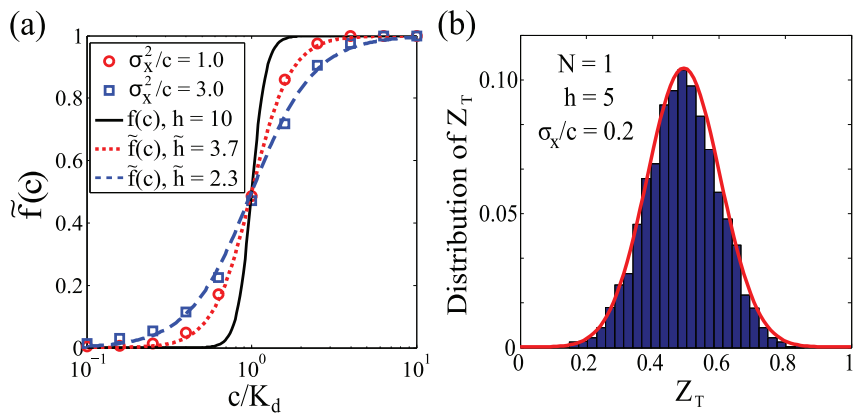

FIG. 1. (Color online) (a) The input-output relationship $\left\langle Y_{i}\right\rangle$ versus $\langle X\rangle=c$. The output $\left\langle Y_{i}\right\rangle$ obtained from simulations (symbols) can be approximated by the Hill function $\widetilde{f}(c)$ with the apparent Hill coefficient $\widetilde{h}$ depending on the Fano factor $\sigma_{x}^{2} / c$. (b) The distribution of $Z_{T}$ in a simulation with $\tau_{y}=1, \tau_{x}=10$, and $T=100$. The solid red line is the fitting of a Gaussian distribution (with standard deviation 0.11).

Fig. 1(a). Nonetheless, the input-output relationship can still be approximated by a new Hill equation:

$$
\left\langle Y_{i}\right\rangle \equiv \widetilde{f}(c) \approx \frac{c^{\widetilde{h}}}{c^{\widetilde{h}}+K_{d}^{\widetilde{h}}},
$$

where the apparent Hill coefficient $\widetilde{h}$ is the observed sensitivity that is less than the intrinsic Hill coefficient $h$. Numerically, it is found that the value of $\widetilde{h}$ decreases with the Fano factor $\sigma_{x}^{2} / c$ [Fig. 1(a)]. For a given Fano factor (fixed $\beta$ ), $\widetilde{h}$ increase with $h$ but tends to saturate as $h \rightarrow \infty$. We will return to explore this issue later on.

\section{RESULTS}

\section{A. Bounds on the output noise}

Many biological sensing functions can be translated into the task of inferring the mean input level $c$ from the observable output signal $\left\{Y_{1}(t), \ldots, Y_{N}(t)\right\}$ over a finite time $T$. The statistical quantity of interest is:

$$
Z_{T} \equiv \frac{1}{T} \int_{0}^{T}\left[\frac{1}{N} \sum_{i=1}^{N} Y_{i}(t)\right] d t,
$$

which converges to a Gaussian distribution by the law of large numbers [Fig. 1(b)]. So we need to consider only the first two moments of $Z_{T}$. As sensors are identical, we expect that $\left\langle Z_{T}\right\rangle=\left\langle Y_{i}\right\rangle=\widetilde{f}(c)$, with the variance $\sigma_{y}^{2} \equiv\left\langle Y_{i}, Y_{i}\right\rangle=$ $\tilde{f}(c)[1-\tilde{f}(c)]$. As different sensors experience the same input fluctuations, their switching events are correlated. We define the covariance $\sigma_{f}^{2} \equiv\left\langle Y_{i}(t), Y_{j}(t)\right\rangle$ for $i \neq j$ and the correlation coefficient $\rho \equiv \sigma_{f}^{2} / \sigma_{y}^{2}$. With the notation $\Delta t=t-t^{\prime}$, we can evaluate the variance of $Z_{T}$ as follows,

$$
\begin{aligned}
\sigma_{z, T}^{2} & \equiv \frac{1}{T^{2} N^{2}} \int_{0}^{T} d t \int_{0}^{T} d t^{\prime} \sum_{i=1}^{N} \sum_{j=1}^{N}\left\langle Y_{i}(t), Y_{j}\left(t^{\prime}\right)\right\rangle \\
& =\frac{\sigma_{y}^{2}}{N T^{2}} \iint\left[\mathcal{A}_{y}(\Delta t)+(N-1) \mathcal{C}_{y}(\Delta t)\right] d t d t^{\prime} \\
& \approx \frac{1}{N} \sigma_{y}^{2} \xi_{y}^{T}+\frac{N-1}{N} \sigma_{f}^{2} \xi_{x}^{T},
\end{aligned}
$$



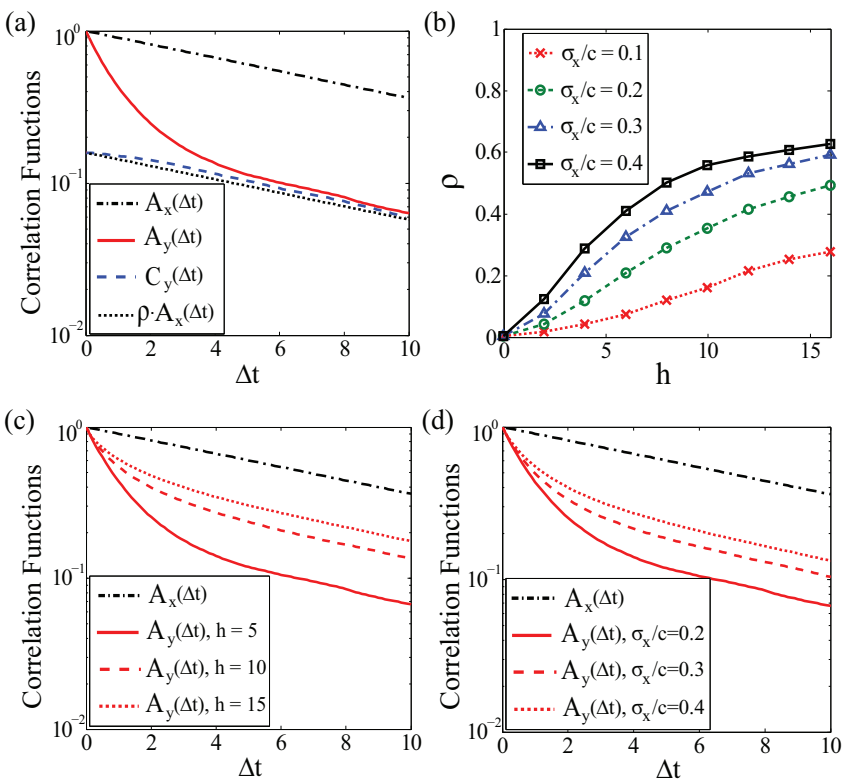

FIG. 2. (Color online) (a) The correlation functions $\mathcal{A}_{y}, \mathcal{C}_{y}$, and $\mathcal{A}_{x}$ versus the time lag $\Delta t$. Parameters used in this simulation: $h=5, c=K_{d}, \sigma_{x} / c=0.2, \tau_{y}=1$, and $\tau_{x}=10$. (b) The correlation coefficient $\rho$ versus the Hill coefficient $h$ for different levels of input noise $\sigma_{x} / c$. (c) $\mathcal{A}_{y}<\mathcal{A}_{x}$, regardless of $h$. (d) $\mathcal{A}_{y}<\mathcal{A}_{x}$, regardless of $\sigma_{x} / c$.

where $\quad \mathcal{A}_{y}(\Delta t) \equiv\left\langle Y_{i}(t), Y_{i}\left(t^{\prime}\right)\right\rangle / \sigma_{y}^{2} \quad$ and $\quad \mathcal{C}_{y}(\Delta t) \equiv$ $\left\langle Y_{i}(t), Y_{j}\left(t^{\prime}\right)\right\rangle / \sigma_{y}^{2}$ denote the normalized autocorrelation and cross-correlation functions. In the last step of Eq. (6), we have introduced the following time-averaging factors:

$$
\begin{aligned}
& \xi_{x}^{T} \equiv \frac{1}{T^{2}} \int_{0}^{T} d t \int_{0}^{T} d t^{\prime} \mathcal{A}_{x}\left(t-t^{\prime}\right), \\
& \xi_{y}^{T} \equiv \frac{1}{T^{2}} \int_{0}^{T} d t \int_{0}^{T} d t^{\prime} \mathcal{A}_{y}\left(t-t^{\prime}\right),
\end{aligned}
$$

and used the approximation, $\mathcal{C}_{y}(\Delta t) \approx \rho \cdot \mathcal{A}_{x}(\Delta t)$, with the insight that the output sensors should become uncorrelated as the input signal loses memory over time. This approximation has been verified by simulating the time traces of two sensors and calculating their correlation functions [Fig. 2(a)]. The correlation coefficient $\rho$ between the output traces increases with the intrinsic sensitivity $h$ and the relative noise level $\sigma_{x} / c$ [Fig. 2(b)]. This can be seen by the assumption that all the receptors see the same slowly fluctuating input and by the use of small noise approximation: $\left\langle Y_{i}(t), Y_{j}(t)\right\rangle=$ $\left\langle f(X)^{2}\right\rangle-\langle f(X)\rangle^{2} \approx\left[f^{\prime}(c)\right]^{2} \sigma_{x}^{2}$, which leads to

$$
\rho=\frac{\sigma_{f}^{2}}{\sigma_{y}^{2}} \approx \frac{\left[f^{\prime}(c)\right]^{2} \sigma_{x}^{2}}{\sigma_{y}^{2}}=h^{2} \frac{f(c)^{2}[1-f(c)]^{2}}{\sigma_{y}^{2}}\left(\frac{\sigma_{x}}{c}\right)^{2} .
$$

Accordingly, Eq. (6) may be approximated as

$$
\sigma_{z, T}^{2} \approx \frac{\sigma_{y}^{2}}{N} \xi_{y}^{T}+\frac{N-1}{N} h^{2} f(c)^{2}[1-f(c)]^{2}\left(\frac{\sigma_{x}}{c}\right)^{2} \xi_{x}^{T},
$$

which is similar to the additive noise rule obtained in the previous literature $[17,18,20]$. However, it is worth noting that $\rho$ is strictly bounded by one and cannot always scale as $h^{2}$ or $\left(\sigma_{x} / c\right)^{2}$, as shown in Fig. 2(b). Thus, Eq. (10) tends to overestimate the effect of input noise in ultrasensitive systems and even explodes when $h \rightarrow \infty$.

The input $X(t)$ has an exponential $\mathcal{A}_{x}$ with correlation time $\tau_{x}$. This allows us to calculate that

$$
\xi_{x}^{T}=2 \frac{\tau_{x}}{T}\left[1-\frac{\tau_{x}}{T}\left(1-e^{-T / \tau_{x}}\right)\right] .
$$

For $T \gg \tau_{x}$, we have $\xi_{x}^{T} \approx 2 \tau_{x} / T$. Though there may be no analytical expression for $\xi_{y}^{T}$, we can find its bounds. As shown in Fig. 2(a), the correlation functions satisfy a general inequality: $\mathcal{C}_{y}<\mathcal{A}_{y}<\mathcal{A}_{x}$ for any time lag $\Delta t$. It is intuitive that the autocorrelation should always be larger than the cross correlation (i.e., $\mathcal{A}_{y}>\mathcal{C}_{y}$ ). The relation $\mathcal{A}_{y}<\mathcal{A}_{x}$ follows from the condition $\tau_{y} \ll \tau_{x}$ and is valid regardless of the sensitivity $h$ or the noise level $\sigma_{x} / c$ [Figs. 2(c)-2(d)]: $\mathcal{A}_{y}$ is dominated by the sensor's intrinsic time $\tau_{y}$ over short time scales and by the input correlation time $\tau_{x}$ over long time scales; for $\Delta t \gg \tau_{y}, \mathcal{A}_{y}(\Delta t)$ decays exponentially at the same rate as $\mathcal{A}_{x}$ does [Fig. 2(a)]. Since $\mathcal{C}_{y} \approx \rho \cdot \mathcal{A}_{x}$, the above inequality implies that $\rho \cdot \mathcal{A}_{x}<\mathcal{A}_{y}<\mathcal{A}_{x}$ and therefore $\rho \cdot \xi_{x}^{T}<\xi_{y}^{T}<\xi_{x}^{T}$. As a result, the variance $\sigma_{z, T}^{2}$ in Eq. (6) must satisfy

$$
\sigma_{y}^{2} \xi_{x}^{T} \rho<\sigma_{z, T}^{2}<\sigma_{y}^{2} \xi_{x}^{T} \frac{1+(N-1) \rho}{N} .
$$

This is one of our key results for biochemical sensing. Intuitively, as correlations between receptors arise only from the input fluctuations, the total noise of sensors should be limited by the input correlation property $\left(\xi_{x}^{T}\right)$. Moreover, the more correlated the receptors are (i.e., larger $\rho$ ), the less efficient they are in averaging out the output noise. In the absence of input noise $(\rho=0)$, Eq. (12) reduces to $0<\sigma_{z, T}^{2}<\sigma_{y}^{2} \xi_{x}^{T} / N$, indicating that $\sigma_{z, T}^{2}$ could be arbitrarily small as $N \rightarrow \infty$. However, the presence of input noise will induce correlations between sensors and thus reduce the capability of population averaging, leading to a lower bound on the output noise. As $\rho \rightarrow 1$, all the sensors perfectly synchronize their switchings and Eq. (12) suggests that $\sigma_{z, T}^{2} \rightarrow \sigma_{y}^{2} \xi_{x}^{T}$, independent of $N$. We can define the effective number of sensors,

$$
N_{\text {eff }} \equiv \frac{N}{1+(N-1) \rho},
$$

which increases with $N$ but saturates $\left(N_{\text {eff }} \leqslant 1 / \rho\right)$. Obviously, the lower bound in Eq. (12) is achieved as $N \rightarrow \infty$. To verify the upper bound, we numerically generate thousands of sample points of $Z_{T}$ for various parameter sets. The sample variance $\sigma_{z, T}^{2}$ for different $N, h$, or $\sigma_{x} / c$ is indeed within the theoretical bounds and tends to saturate as the input noise increases (Fig. 3).

We can derive an intuitive understanding of Eq. (12) by considering the limit $\tau_{y} \rightarrow 0$, under which the sensors switch extremely fast such that the state of each sensor at any time can be regarded as a Bernoulli random variable:

$$
Y_{i}(t)=\left\{\begin{array}{lll}
1, & \text { with probability } & f(X(t)), \\
0, & \text { with probability } & 1-f(X(t)),
\end{array}\right.
$$

where $f(X(t))$ denotes the instantaneous probability to find the $i$ th sensor being in the on state at time $t$. In this case, 
(a)

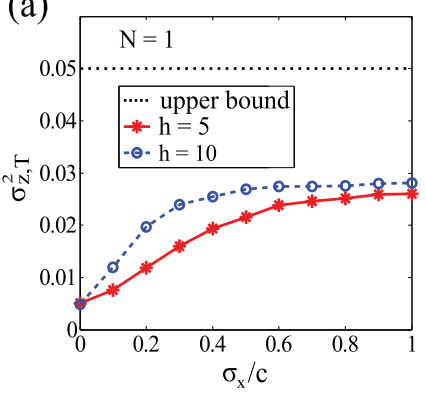

(b)

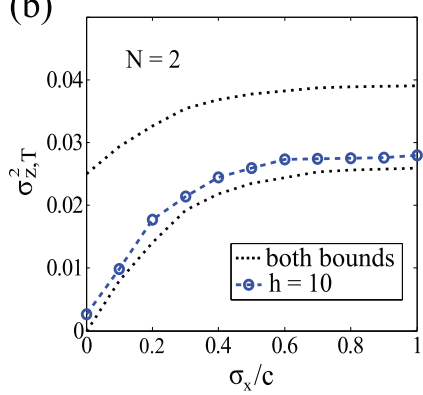

FIG. 3. (Color online) (a) $\sigma_{z, T}^{2}$ versus $\sigma_{x} / c$ for a single sensor with $h=5$ and $h=10$. In these simulations, we used $\tau_{y}=1, \tau_{x}=$ 10 , and $T=100$. By Eq. (12), the upper bound of $\sigma_{z, T}^{2}$ for $N=1$ is $\sigma_{y}^{2} \xi_{x}^{T} \approx 0.05$ at $c=K_{d}$. (b) $\sigma_{z, T}^{2}$ versus $\sigma_{x} / c$ for two sensors $(N=2)$ with $h=10$ at $c=K_{d}$. Here, the upper and lower bounds of $\sigma_{z, T}^{2}$ are $\sigma_{y}^{2} \xi_{x}^{T}(1+\rho) / 2$ and $\sigma_{y}^{2} \xi_{x}^{T} \rho$, respectively. Both bounds (dotted lines) depend on the correlation coefficient $\rho$ which is shown in Fig. 2(b).

$\rho=\sigma_{f}^{2} / \sigma_{y}^{2}$ represents the instantaneous correlation coefficient between two sensors. The variance of $Z(t) \equiv \frac{1}{N} \sum_{i=1}^{N} Y_{i}(t)$ is found to be:

$$
\sigma_{z}^{2}=\frac{1}{N} \sigma_{y}^{2}+\frac{N-1}{N} \sigma_{f}^{2}=\sigma_{y}^{2} \frac{1+(N-1) \rho}{N} .
$$

As $\tau_{z} \approx \tau_{x}$ when $\tau_{y} \rightarrow 0$, we have $\sigma_{z, T}^{2} \approx \sigma_{z}^{2} \frac{2 \tau_{z}}{T} \approx \sigma_{z}^{2} \xi_{x}^{T}$ which recovers the upper bound in Eq. (12).

\section{B. Limit on the apparent sensitivity}

As we mentioned before, in the presence of input noise, the apparent Hill coefficient $(\widetilde{h})$ increases with the intrinsic Hill coefficient $(h)$ and tends to saturate as $h \rightarrow \infty$. Indeed, there exists a limit on $\widetilde{h}$, denoted by $\widetilde{h}_{\infty}$, which can be obtained by taking the limit $h \rightarrow \infty$ and $\tau_{y} \rightarrow 0$. In this scenario, every sensor behaves as an indicator function:

$$
Y_{i}(t)=\left\{\begin{array}{lll}
1 & \text { if } \quad X(t) \geqslant K_{d} \\
0 & \text { if } & X(t)<K_{d}
\end{array}\right.
$$

The expectation of $Y_{i}$ is simply the tail distribution of the gamma probability density Eq. (2), i.e.,

$$
\left\langle Y_{i}\right\rangle \equiv \tilde{f}(c)=\frac{\beta^{\alpha}}{\Gamma(\alpha)} \int_{K_{d}}^{\infty} x^{\alpha-1} e^{-\beta x} d x \equiv \Gamma\left(\alpha, \beta K_{d}\right),
$$

where $\Gamma\left(\alpha, \beta K_{d}\right)$ is also known as the regularized (upper) gamma function. Given an input distribution (fixed $\alpha$ and $\beta$ ), one can change $K_{d}$ to probe the input mean level, $c=\alpha / \beta$, with a sensitivity given by

$$
\left|\frac{\partial \Gamma\left(\alpha, \beta K_{d}\right)}{\partial K_{d}}\right|=\frac{e^{-\beta K_{d}}\left(\beta K_{d}\right)^{\alpha}}{K_{d} \Gamma(\alpha)}=p\left(X=K_{d}\right) .
$$

This function reaches the maximum at $K_{d}^{*}=c-1 / \beta$. Therefore, for $\alpha=\beta c \gg 1$, the sensor is most sensitive near $c=K_{d}$. Alternatively, for a given indicator sensor with fixed $K_{d}$, we can change $c$ (with fixed $\beta$ ) to examine at which level of $c$ the sensor is most sensitive to the input signal. By symmetry, the maximum sensitivity should be achieved around $c^{*}=$ $K_{d}+1 / \beta$. As shown in Fig. 4(a), the input-output relationship
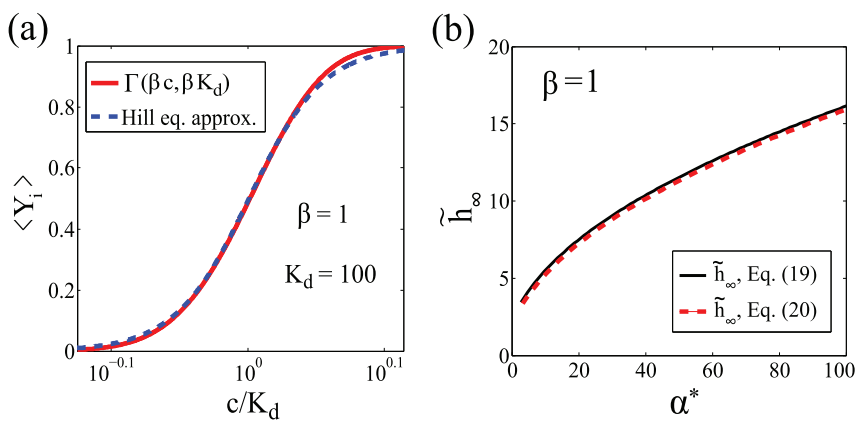

FIG. 4. (Color online) (a) The input-output relationship, $\left\langle Y_{i}\right\rangle=$ $\Gamma\left(\beta c, \beta K_{d}\right)$, can be approximated by the Hill equation, $c^{\widetilde{h}_{\infty}} /\left(c^{\widetilde{h}_{\infty}}+\right.$ $K_{d}^{\widetilde{h}_{\infty}}$ ), with $\widetilde{h}_{\infty}$ given by Eq. (19). (b) The limit Hill coefficient, $\widetilde{h}_{\infty}$, as a function of the signal-to-noise ratio $\alpha^{*}=\beta c^{*}=\left(c^{*} / \sigma_{x}\right)^{2}$ at $c^{*}=K_{d}+1 / \beta$ for fixed $\beta=1$.

$\tilde{f}(c)=\Gamma\left(\beta c, \beta_{\tilde{n}} K_{d}\right)$ can be approximated by a Hill equation, $c^{\widetilde{h}_{\infty}} /\left(c^{\widetilde{h}_{\infty}}+K_{d}^{\widetilde{h}_{\infty}}\right)$, with the limit Hill coefficient given by

$$
\widetilde{h}_{\infty}=4\left|\frac{\partial \Gamma\left(\beta c, \beta K_{d}\right)}{\partial \log \left(K_{d}\right)}\right|_{c=c^{*}}=\frac{4 e^{-\beta K_{d}}\left(\beta K_{d}\right)^{\beta K_{d}}}{\Gamma\left(\beta K_{d}\right)} .
$$

Clearly, $\widetilde{h}_{\infty}$ is determined by both the scale parameter $\beta$ of the input distribution and the location parameter $K_{d}$ of the sensor [Fig. 4(b)]. The factor of 4 in Eq. (19) arises from the fact that the maximum of the derivative of a Hill equation (with the Hill coefficient $h$ ) is given by $h / 4$. The input signal-to-noise ratio at $c^{*}$ is $\alpha^{*}=\beta c^{*}=\left(c^{*} / \sigma_{x}\right)^{2}$. So we have that $\beta K_{d}=\beta c^{*}-1=$ $\alpha^{*}-1$. Using the asymptotic formula $\Gamma(x) \approx \sqrt{2 \pi} e^{-x} x^{x-\frac{1}{2}}$ for $x \gg 1$, we can rewrite Eq. (19) as

$$
\widetilde{h}_{\infty} \approx 4 \sqrt{\frac{\beta K_{d}}{2 \pi}}=\sqrt{\frac{8\left(\alpha^{*}-1\right)}{\pi}},
$$

which increases with the signal-to-noise ratio $\alpha^{*}$ of the input near $c=K_{d}$ [Fig. 4(b)]. The asymptotic scaling, $\widetilde{h}_{\infty} \sim \sqrt{\alpha^{*}}=$ $c^{*} / \sigma_{x}$ by Eq. (20), suggests that $\widetilde{h}_{\infty}$ is constrained by the relative intensity of input noise.

The above analysis suggests that, for any ultrasensitive sensors, the apparent sensitivity $\widetilde{h}$ read from the input-output response is always bounded by the limit Hill coefficient, i.e., $\widetilde{h}<\widetilde{h}_{\infty}$. We have numerically tested this inequality in various parameter regimes and found that $\widetilde{h}<\widetilde{h}_{\infty}$ holds in general [Fig. 5(a)]. It is also worth remarking that the above argument or conclusion does not depend on how we model the input process. The general limit $\widetilde{h}<\widetilde{h}_{\infty}$ can be similarly obtained when assuming a different input distribution (e.g., a Poisson distribution).

\section{General limit to biochemical sensing}

The mean input level $c$ is encoded by the output sensory signal. By the law of large numbers, the statistic $\bar{Z}_{T}$ should converge to $\left\langle\bar{Z}_{T}\right\rangle=\tilde{f}(c) \approx c^{\widetilde{h}} /\left(c^{\widetilde{h}}+K_{d}^{\widetilde{h}}\right)$. Thus, a good estimator of $c$ can be found by inverting $\bar{Z}_{T}=\widetilde{f}(c)$. When the integration time $T$ is sufficiently large, the output noise $\sigma_{z, T}^{2}$ can be small enough so that one can use the error propagation formula to examine the accuracy $(\delta c / c)$ of this estimator. By 
(a)

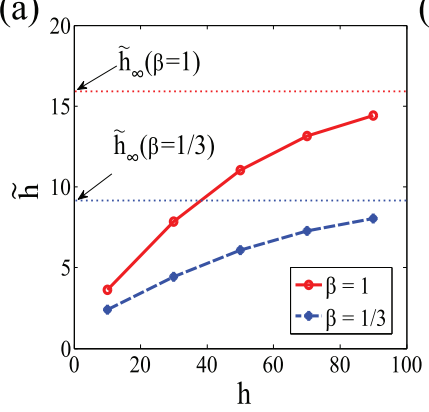

(b)

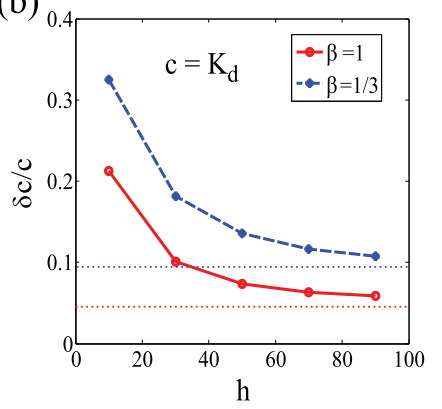

FIG. 5. (Color online) (a) $\tilde{h}$ versus $h$ for two different values of $\beta: \beta=1$ (solid red line) and $\beta=1 / 3$ (dashed blue line). (b) $\delta c / c$ versus $h$ at $c=K_{d}$ for $\beta=1$ (solid red line) and $\beta=1 / 3$ (dashed blue line). The dotted lines correspond to the limit values of $\widetilde{h}$ in (a) and $\delta c / c$ in (b) under the limit $h \rightarrow \infty$. We have used $K_{d}=100$, $\tau_{y}=1, \tau_{x}=10$, and $T=100$ in numerical simulations.

further using Eq. (12), we have that

$$
\frac{\xi_{x}^{T} \rho}{\widetilde{h}^{2} \sigma_{y}^{2}}<\left(\frac{\delta c}{c}\right)^{2}=\frac{\sigma_{z, T}^{2}}{c^{2}}\left[\frac{d \widetilde{f}}{d x}(c)\right]^{-2}<\frac{\xi_{x}^{T}}{\widetilde{h}^{2} \sigma_{y}^{2} N_{\mathrm{eff}}},
$$

where $\sigma_{y}^{2} \approx\left(c K_{d}\right)^{\widetilde{h}} /\left(c^{\widetilde{h}}+K_{d}^{\widetilde{h}}\right)^{2}$. Equation (21) shows that ultrasensitivity $(\widetilde{h})$ could help the system read out the input level. However, this effect is limited due to the constraint $\widetilde{h}<\tilde{\widetilde{h}}_{\infty}$. Interestingly, increasing the intrinsic Hill coefficient $h$ increases the sensitivity $(\widetilde{h})$, which tends to reduce the sensing error, but with fluctuations in the input it also increases the correlations between receptors $(\rho)$ and hence decrease the effective number of sensors $\left(N_{\text {eff }}\right)$, which tends to raise the sensing error. This tradeoff demonstrates the interplay between the sensitivity and the efficiency of sensors when the input is noisy. From numerical experiments, it is also found that the sensing error overall decreases with increasing $h$ but saturates as $h \rightarrow \infty$, as can be seen in Fig. 5(b).

Is there some fundamental limit to biochemical sensing? If the input is directly observable and we estimate the mean concentration from the sample $X(t)$ over a time window $T$, then the total variance is $\sigma_{x, T}^{2}=\sigma_{x}^{2} \xi_{x}^{T}$ and the sensing limit is $(\delta c / c)^{2}=\sigma_{x, T}^{2} / c^{2}=\xi_{x}^{T}\left(\sigma_{x} / c\right)^{2}$. If $X(t)$ is not directly observable and the mean level $c$ has to be inferred from some output, then the actual sensing error must be larger than the sensing limit, i.e.,

$$
\left(\frac{\delta c}{c}\right)^{2}>\left(\frac{\sigma_{x}}{c}\right)^{2} \xi_{x}^{T}=\frac{\xi_{x}^{T}}{\beta c} .
$$

This inequality sets the lower bound for the measurement error in biological sensing systems. This lower bound is solely determined by the input properties $\left(\sigma_{x}, c\right.$, and $\left.\xi_{x}^{T}\right)$. In fact, the general inequality Eq. (22) can easily recover the Berg-Purcell limit. When $X(t)$ refers to the number of molecules diffusing (with diffusion coefficient $D$ ) in an open volume of radius $R$, the input correlation time is [4]: $\tau_{x}=\frac{2}{5} R^{2} / D$, which is the typical time for molecules within the volume to be renewed by diffusion. For $T \gg \tau_{x}$, we also have $\xi_{x}^{T} \approx 2 \tau_{x} / T$. The number of molecules in this volume is a Poisson random variable,

satisfying $\left(\sigma_{x} / c\right)^{2}=1 / c$ where $c \equiv 4 \pi R^{3} \bar{c} / 3$ is the average number of molecules in the volume with $\bar{c}$ denoting the mean concentration. Plugging all these results into Eq. (22) leads to the following inequality,

$$
\left(\frac{\delta c}{c}\right)^{2}>\frac{3}{4 \pi R^{3} \bar{c}} \cdot \frac{4 R^{2}}{5 D T}=\frac{3}{5 \pi D R \bar{c} T},
$$

which recovers the Berg-Purcell limit for the perfect monitor that counts the number of molecules inside itself [4]. According to Eq. (23), no matter how many or how sensitive receptors are used, the sensing error is always bounded from below by the counting noise. In fact, one can view Eq. (22) as a generalized form of the Berg-Purcell limit, as it can be applied to both Poisson and non-Poisson process.

Unlike the perfect monitor, an ultrasensitive sensor usually responds only to a narrow range of the input signal. Such sensors are able to provide accurate measurements near $K_{d}$, but become insensitive to the stimuli away from $K_{d}$. The BergPurcell limit does not capture such locally sensitive property, whereas our Eq. (21) does. One can obtain valuable insights by considering the extreme scenario where each sensor behaves as an indicator function Eq. (16). In this limit, we have $\rho \rightarrow 1$, $N_{\text {eff }} \rightarrow 1$, and $\widetilde{h} \rightarrow \widetilde{h}_{\infty}$ such that Eq. (21) becomes

$$
\left(\frac{\delta c}{c}\right)^{2}=\frac{\xi_{x}^{T}}{\widetilde{h}_{\infty}^{2} \sigma_{\infty}^{2}},
$$

where $\sigma_{\infty}^{2}=\Gamma\left(\beta c, \beta K_{d}\right)\left[1-\Gamma\left(\beta c, \beta K_{d}\right)\right]$. For $\beta c \gg 1$, we have $\sigma_{\infty}^{2} \approx \frac{1}{4}$ at $c=K_{d}$.

Comparing Eq. (24) with Eq. (22), one can see that if $\widetilde{h}_{\infty}$ were larger than $2 \sqrt{\beta K_{d}}$, then the sensing limit given by Eq. (24) would be able to beat the fundamental limit Eq. (22) at $c=K_{d \dot{\tilde{h}}}$ However, using Eq. (19), one can verify that the inequality $\widetilde{h}_{\infty}<2 \sqrt{\beta K_{d}}$ always holds for $\beta K_{d}>1$. By Eq. (20), we have that

$$
\frac{2 \sqrt{\beta K_{d}}}{\widetilde{h}_{\infty}} \approx \sqrt{\frac{\pi}{2}} \approx 1.2533 .
$$

In other words, the sensing error $(\delta c / c)$ by an indicator sensor near $K_{d}$ is roughly $25 \%$ higher than the sensing limit of a perfect monitor. This can be understood as the information gained by an infinitely sensitive receptor $(h \rightarrow \infty)$ is still less than the information collected by the perfect monitor, which records everything.

The above arguments lead to a new sensing limit near $K_{d}$ for ultrasensitive systems:

$$
\left(\frac{\delta c}{c}\right)^{2}>\frac{\xi_{x}^{T}}{\widetilde{h}_{\infty}^{2} \sigma_{\infty}^{2}} \approx \frac{\pi \xi_{x}^{T}}{8 \beta K_{d} \Gamma\left(\beta c, \beta K_{d}\right)\left[1-\Gamma\left(\beta c, \beta K_{d}\right)\right]} .
$$

Figure 6 gives a numerical comparison of the lower bound of $\delta c / c$ in Eq. (26) to that in Eq. (22). One can see that our newly derived sensing limit (dashed red line) is strictly larger than the Berg-Purcell limit (solid blue line). Interestingly, the presence of input noise helps increase the dynamic range of sensing: without the input noise, the ultrasensitive receptor would be very precise at $c=K_{d}$, but almost useless elsewhere; the presence of noise effectively increases the accuracy of 


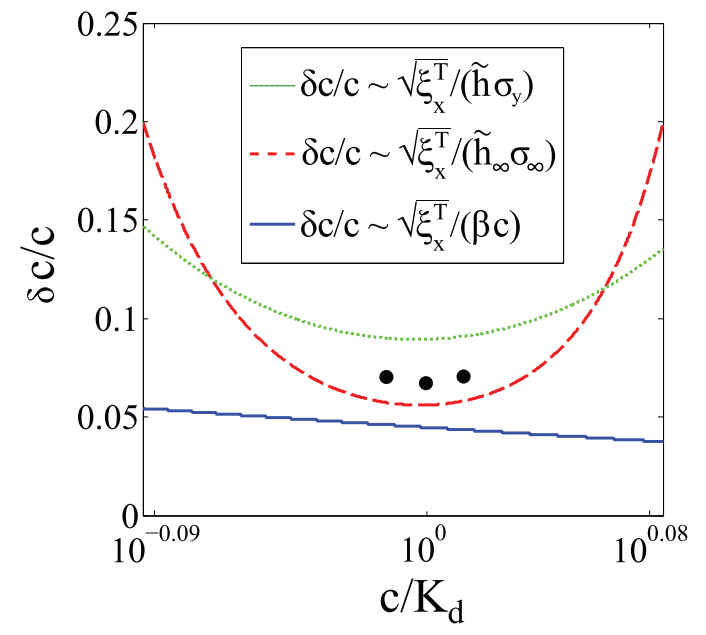

FIG. 6. (Color online) A numerical comparison between the lower bound (solid blue line) in Eq. (22) and the lower bound (dashed red line) in Eq. (26). The dotted green line corresponds to the upper bound of $\delta c / c$ in Eq. (21) for a single sensor $(N=1)$ with $\widetilde{h}=10$. The black symbols represent the Monte Carlo simulation results of $\delta c / c$ by a sensor with the apparent sensitivity $\widetilde{h} \approx 10$ under the Poisson noise (i.e., $\beta=1$ ) at different mean concentration levels. The sensing error $\delta c / c$ was calculated using the error propagation formula in Eq. (21). These black symbols fall between the bounds as indicated by Eq. (27). Other parameters used here include $K_{d}=100$, $\tau_{y}=1, \tau_{x}=10$, and $T=100$.

signaling in the neighborhood of $K_{d}$. We also plot in Fig. 6 the upper bound (dotted green line) of $\delta c / c$ given by Eq. (21) for a single sensor $(N=1)$ with the apparent Hill coefficient $\widetilde{h}$. The key implication of the above theoretical arguments is that, for a sensor with known $K_{d}$ and the apparent sensitivity $\widetilde{h}$, its accuracy of biochemical measurements near $K_{d}$ should be bounded as follows:

$$
\frac{\sqrt{\xi_{x}^{T}}}{\widetilde{h}_{\infty} \sigma_{\infty}}<\frac{\delta c}{c}<\frac{\sqrt{\xi_{x}^{T}}}{\widetilde{h} \sigma_{y}} .
$$

The above simple inequality has been tested by numerical simulations (black symbols in Fig. 6).

\section{CONCLUSION}

In conclusion, we have presented results, both analytical and numerical, for biochemical sensing in ultrasensitive systems. First, we demonstrate that biochemical noise does not accumulate additively but is largely limited by the noise-induced correlated property of ultrasensitive sensors. This contrasts with previous belief from the linear-noise approximation, which tends to exaggerate the impact of the input noise. Second, we show that the input noise also constrains the apparent sensitivity of the system. Thus, although ultrasensitivity is able to improve the sensing accuracy, it cannot beat the physical limit set by the molecular counting noise. Finally, our analysis leads to a sensing limit for ultrasensitive systems, which is more appropriate for ultrasensitive signaling systems. In sum, the results we present in this paper provide insights about the interplay between noise and ultrasensitivity in biochemical signal detection.

\section{ACKNOWLEDGMENTS}

We would like to thank Yuhai Tu, David A. Kessler, and two anonymous referees for all the valuable comments in the earlier stage of this work. This work was supported by the National Science Foundation (NSF) Grant No. DMS-1068869, by the National Institutes of Health (P01 GM078586), and by the NSF Center for Theoretical Biological Physics (Grant No. NSF PHY-1308264). H.L. was also supported by the CPRIT Scholar program of the State of Texas.
[1] P. Cluzel, M. Surette, and S. Leibler, Science 287, 1652 (2000).

[2] C. Y. Huang and J. E. Ferrell, Proc. Natl. Acad. Sci. USA 93, 10078 (1996).

[3] J. E. Ferrell and E. M. Machleder, Science 280, 895 (1998).

[4] H. C. Berg and E. M. Purcell, Biophys. J. 20, 193 (1977).

[5] W. Bialek and S. Setayeshgar, Proc. Natl. Acad. Sci. USA 102, 10040 (2005).

[6] W. Bialek and S. Setayeshgar, Phys. Rev. Lett. 100, 258101 (2008).

[7] K. Wang, W.-J. Rappel, R. Kerr, and H. Levine, Phys. Rev. E 75, 061905 (2007).

[8] W.-J. Rappel and H. Levine, Phys. Rev. Lett. 100, 228101 (2008).

[9] R. G. Endres and N. S. Wingreen, Proc. Natl. Acad. Sci. USA 105, 15749 (2008).

[10] B. Hu, W. Chen, W.-J. Rappel, and H. Levine, Phys. Rev. Lett. 105, 048104 (2010).

[11] B. Hu, W. Chen, W.-J. Rappel, and H. Levine, Phys. Rev. E 83, 021917 (2011).

[12] T. Mora and N. S. Wingreen, Phys. Rev. Lett. 104, 248101 (2010).
[13] M. Skoge, Y. Meir, and N. S. Wingreen, Phys. Rev. Lett. 107, 178101 (2011).

[14] R. G. Endres and N. S. Wingreen, Phys. Rev. Lett. 103, 158101 (2009).

[15] C. C. Govern and P. R. ten Wolde, Phys. Rev. Lett. 109, 218103 (2012).

[16] K. Kaizu, W. de Ronde, J. Paijmans, K. Takahashi, F. Tostevin, and P. R. ten Wolde, Biophys. J. 106, 976 (2014).

[17] T. Shibata and K. Fujimoto, Proc. Natl. Acad. Sci. USA 102, 331 (2005).

[18] S. Tănase-Nicola, P. B. Warren, and P. R. ten Wolde, Phys. Rev. Lett. 97, 068102 (2006).

[19] T. Lu, M. Ferry, R. Weiss, and J. Hasty, Phys. Biol. 5, 036006 (2008).

[20] J. Paulsson, Nature (London) 427, 415 (2004).

[21] B. Hu, D. A. Kessler, W.-J. Rappel, and H. Levine, Phys. Rev. E 86, 061910 (2012).

[22] H. Park, P. Oikonomou, C. C. Guet, and P. Cluzel, Biophys. J. 101, 2336 (2011).

[23] B. Hu and Y. Tu, Phys. Rev. Lett. 110, 158703 (2013).

[24] B. Hu, D. A. Kessler, W.-J. Rappel, and H. Levine, Phys. Rev. Lett. 107, 148101 (2011). 